\title{
A COMPARISON OF THE ROLE OF THE BRONCHIAL ARTERIES IN BRONCHIECTASIS AND IN EXPERIMENTAL LIGATION OF THE PULMONARY ARTERY
}

\author{
BY \\ F. B. COCKETT AND C. C. N. VASS \\ From the Departments of Surgery and Physiology, St. Thomas's Hospital, London
}

(RECEIVED FOR PUBLICATION JUNE 5, 1951)

The hypertrophy of the bronchial arteries in bronchiectasis has been known to surgeons ever since resection of the lung had been practised for this condition. Histologists have noticed and commented upon the extreme vascularity of the tissue round the bronchiectatic sacs, and have tried to explain this as part of the general inflammatory change. The physiological importance and significance of this bronchial artery hypertrophy have only recently been appreciated. By means of perfusion experiments, followed by the preparation of complete vascular casts in neoprene, it has been possible to demonstrate accurately the anatomical extent of this hypertrophy, and to show that an extensive anastomosis develops between the bronchial arteries and the pulmonary arteries in these lungs. Moreover, the vascular pathology is comparable to that which occurs in the experimental dog after ligation of the pulmonary artery. This is a surprising observation, as the stimulus to the development of the bronchial collateral circulation appears to be so dissimilar in the two cases.

\section{EXPERIMENTAL WORK}

The original reason for beginning the experimental work was to show that if the pulmonary artery to a lung were tied, a collateral blood supply could be directed into it by creating adhesions between it and the chest wall. It was found that branches from the intercostals entered the adhesions and anastomosed with the hypertrophied subpleural capillary network, which was supplied mainly by the enlarged bronchial arterial system within the lung. It was while studying this collateral circulation that our attention was drawn to the hypertrophy of these bronchial vessels, and to the fact that they ramified throughout the lung as large tortuous channels almost as big as the original pulmonary arteries. Moreover, it was evident that communications had developed between these enlarged bronchial arteries and the pulmonary arteries, which were precapillary in situation. No such precapillary anastomosis between bronchial arteries and pulmonary veins could be 
demonstrated. The state of affairs which we believe to exist in these lungs is summarized by Fig. 1 .

In order to demonstrate more conclusively the extent of the bronchial hypertrophy and the presence of these anastomoses, it was decided to make neoprene casts of the vascular system of lungs whose pulmonary arteries had been tied.

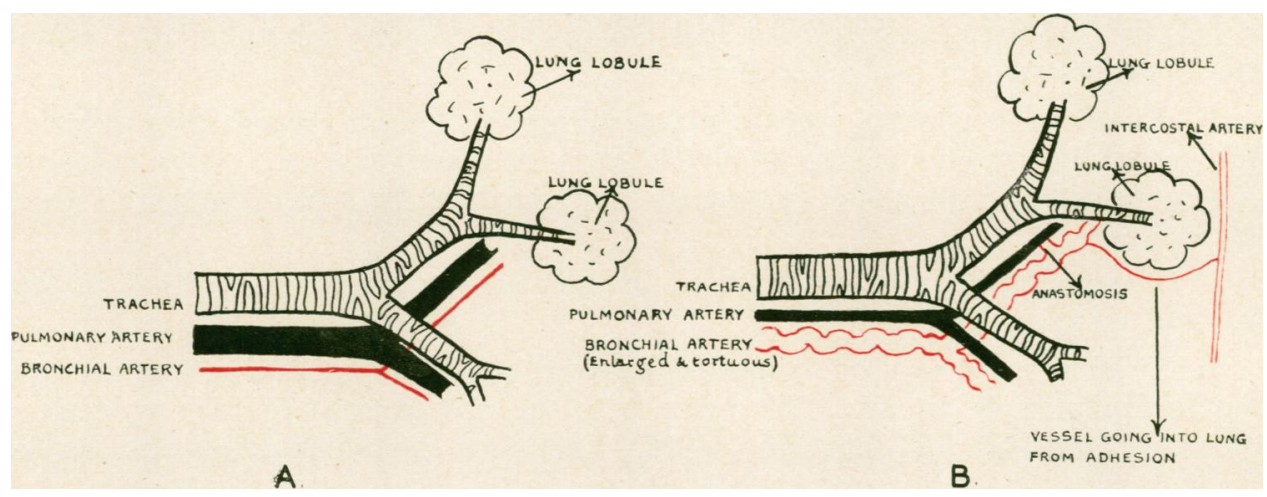

FIG. 1.-A. Conditions in lung whose pulmonary artery has been tied for six to nine months. B. Conditions in normal lung.

\section{TECHNIQUE}

Neoprene is a fluid plastic which sets to a solid rubbery consistence a few hours after contact with animal tissues. It can be obtained in several colours, so that bronchi, bronchial arteries, and pulmonary arteries and veins can all be injected in different colours. The lung to be injected must be from a recently killed animal. The bronchial arteries are injected by isolating the segment of aorta from which they arise and injecting through a cannula tied into this. The pulmonary arteries and veins are easy to cannulate and inject. The bronchi are filled by merely pouring the neoprene into the open end of the trachea. It is difficult to avoid flooding the alveoli with neoprene and thus obscuring the vascular picture by the density of the casts obtained. For this reason we have occasionally refrained from filling the bronchi at all.

A necessary preliminary to the neoprene injection is to flush all the vascular channels with saline, and during this preliminary perfusion of the vessels several pertinent observations were made. The neoprene is then injected, care being taken to exclude all air bubbles. The injection pressure never exceeds $140 \mathrm{~mm}$. $\mathrm{Hg}$. We consider this a most important point, because at pressures higher than this, the systolic arterial pressure of the dog, artifacts due to the high pressure of the injection may be produced. The vessels are then tied off and the whole specimen immersed in $10 \%$ formalin for four days. It is then transferred to concentrated $\mathrm{HCl}$ for 48 hours at a temperature of about $30^{\circ} \mathrm{C}$. This digests away all the tissues, leaving the neoprene casts intact. The cast is difficult to handle, as it is so delicate, and all manipulations and dissections must be done with it floating in water. Small pieces may be isolated and teased out under the dissecting microscope to show the minute anatomy of the blood vessels. It was found that even had they not been injected in different colours it would have been easy to pick out the bronchial arteries and differentiate them from the pulmonary arteries and veins as they are anatomically so distinct. 
In order to preserve specimens or selected parts of them for examination or demonstration purposes, we have mounted them in transparent blocks of " marco-resin." Plates 1,2 , and 3 show specimens mounted in this material. They keep indefinitely and are extremely easy to handle. The technique of this procedure is given at the end of the paper for those interested.

\section{RESULTS}

A study of these casts showed that one year after tying the pulmonary artery the following changes had taken place:

1. There was hypertrophy of the bronchial arteries, which became large, dilated, and extremely tortuous vessels about 10 times their previous size. They followed closely the course of the pulmonary artery and bronchi in the lung. These are shown in Plate 3.

2. The pulmonary artery decreased in size but remained patent distal to the ligature.

3. Visible communications appeared between branches of the bronchial arteries and the pulmonary arteries. We agree with the observations of Liebow, Hales, Bloomer, Harrison, and Lindskog (1950a) that these communications are found at the base of the lobular artery: that is to say, fairly distal in the pulmonary artery tree.

The bronchial collateral develops very quickly. Bloomer, Harrison, Lindskog, and Liebow (1949) have shown that the collateral increases to nearly its maximum size in about three months. A comparison of Plates 1 and 2 shows what can happen in just seven days. This dog was killed and the bronchial arteries to both lungs injected seven days after tying the left pulmonary artery. But a very interesting difference will be noticed between the specimens shown in Plates 2 and 3 . In Plate 3 the whole of the pulmonary artery and lung capillaries have been retrogradely injected via the enlarged bronchial arteries (cast made from the lung of a dog one year after tying the pulmonary artery). In Plate 2, although the bronchial arteries have enlarged, there is no injection of the lung capillaries or pulmonary artery. The answer to this is provided by Plate 4, which shows the bronchopulmonary anastomosis which develops in the course of time, by means of which the pulmonary artery and hence the lung capillaries are filled from the bronchial artery.

One further point may be gleaned from a microscopic study of the specimen shown in Plate 3 . This lung was adherent to the chest wall, and in the adhesions vessels from the intercostals can be seen entering the lung. These vessels anastomose not with lung capillaries or small pulmonary artery branches, but with branches of the enlarged bronchial artery system within the lung. Hence the blood from these vessels can take a very active part in the collateral circulation of the lung, as they have access to the bronchopulmonary communications. The state of affairs in these lungs is therefore well represented by Fig. 1.

\section{StUdies on BRonchiectasis}

Bronchovascular casts were prepared from a case of an adult man suffering from total bronchiectasis of the left lung only. 
The patient, a man aged 40, was first seen in October, 1950 . He complained of a severe productive cough for nine years. He had had several haemoptyses during the past three years, culminating in a large one of approximately half a pint before admission. On examination it was seen that he had gross clubbing of fingers and toes ; flattening of the left hemithorax ; and the signs of complete collapse of the left lung. Radiographs confirmed that the left lung was completely collapsed and bronchiectatic. Pneumonectomy was advised, and was carried out by N. R. Barrett in December, 1950. The patient made a good recovery and has since gone back to work.

The fresh operation specimen was at once perfused with saline by cannulae inserted into the enlarged bronchial artery, the pulmonary artery, and a pulmonary vein. The saline perfusion was started at a pressure of $100 \mathrm{~mm}$. $\mathrm{Hg}$ through the bronchial artery. At once the perfusion fluid welled up in the cannula tied into the pulmonary artery and started to drip from it. Nothing issued from the pulmonary vein cannula unless the pulmonary artery was first occluded. If this was done, there was a delay of about 15 seconds, then the perfusion fluid dripped out of the pulmonary vein cannula rather slowly. It was thus apparent that in this lung there were large communications between the bronchial and pulmonary arteries. The extent of these communications was more convincingly demonstrated if some methylene blue was injected into the bronchial artery perfusion fluid. The effluent from the pulmonary artery became blue within two seconds; it was at least two minutes before any perceptible staining of the general lung tissue was apparent.

The bronchovascular neoprene cast was then made, using red neoprene for the bronchial artery, blue for the pulmonary artery, and white for the bronchus. The dissection of these casts later revealed the following points.

There was, as expected, a great hypertrophy of the bronchial arterial system throughout the lung. The extent of this bronchial collateral circulation can be judged by reference to Plate 5 . The bronchiectatic sacs in particular were surrounded by a vast network of tortuous vessels derived from the bronchial arterial system.

Amongst these bronchial vessels in the walls of the bronchiectatic sacs were numerous examples of aneurysm formation. These are illustrated in Plate 5. Most of them appear to be fusiform dilatations, but several examples of saccular "blowouts" were seen. It would seem reasonable to assume that it is a bursting or erosion of these aneurysms which is responsible for the episodes of haemoptysis which occur in patients with bronchiectasis. These have already been noticed in the sections of bronchiectatic lungs by Tait Smith (1951).

The pulmonary arteries appear shrunken, and the casts of them are often either irregular or show longitudinal furrows. This appearance is consistent with the theory that the smaller pulmonary artery branches become thrombosed in severe bronchiectasis and are recanalized by connexions with the bronchial arteries.

Although red neoprene was originally injected into the bronchial artery and blue into the pulmonary artery, when the casts were examined there was an irregular mixture of red and blue in practically all but the larger vessels. Thus it was not sufficient to say that if a vessel was red it was part of the bronchial artery system, as quite a large number of vessels which were quite obviously bronchial in origin were filled with blue. This phenomenon had been noticed to a lesser extent in the bronchovascular casts of the dogs' lungs as well. The reason for this haphazard 
mixing of colours was presumably the extensive communication between the bronchial arteries and pulmonary arteries in this lung.

These communications, in contrast to those in the dogs' lungs, were large, and many of them were easily visible to the naked eye. They were of two general varieties. Many of the larger ones occurred at the bifurcations of third, fourth, and fifth order branches of the pulmonary artery. A typical anastomosis of this sort is shown in Plate 6. The size of the actual site of anastomosis in this case is nearly $1 \mathrm{~mm}$. in diameter, and there were numerous others as big in this lung. More peripheral pulmonary artery branches had smaller communications going straight into the side as shown in Plate 7. Plate 8 shows some of the very smallest communications taken from the wall of a bronchiectatic sac; these are multiple and only $50-100 \mu$ in size.

\section{Discussion}

The interest which has sprung up in the last few years in the role of the bronchial arteries in disease owes its impetus largely to Blalock and Taussig. The surgical approach to the problems of congenital heart disease at once led to a widespread realization of the importance of the bronchial arteries in the collateral circulation of the lungs in such cases. The efficiency of this naturally occurring collateral circulation was apparent, particularly in patients in whom the absence of the pulmonary artery was complete and were yet able to live. Since then it has been shown that this bronchial collateral circulation develops in a number of chronic fibrotic states of the lung-bronchiectasis, tuberculosis, infarction, and emphysema. Carcinoma tissue in the lung may also derive its blood supply from the bronchial system (Wright, 1938 ; Liebow et al., 1950). The bronchial collateral circulation can be stimulated to develop in the experimental animal by ligating the pulmonary artery, and this has afforded a means of study.

The interesting fact emerges that in every case in which this bronchial collateral has received detailed study it has been found that the development of anastomoses between the enlarged bronchial system and the pulmonary arterial system occurs.
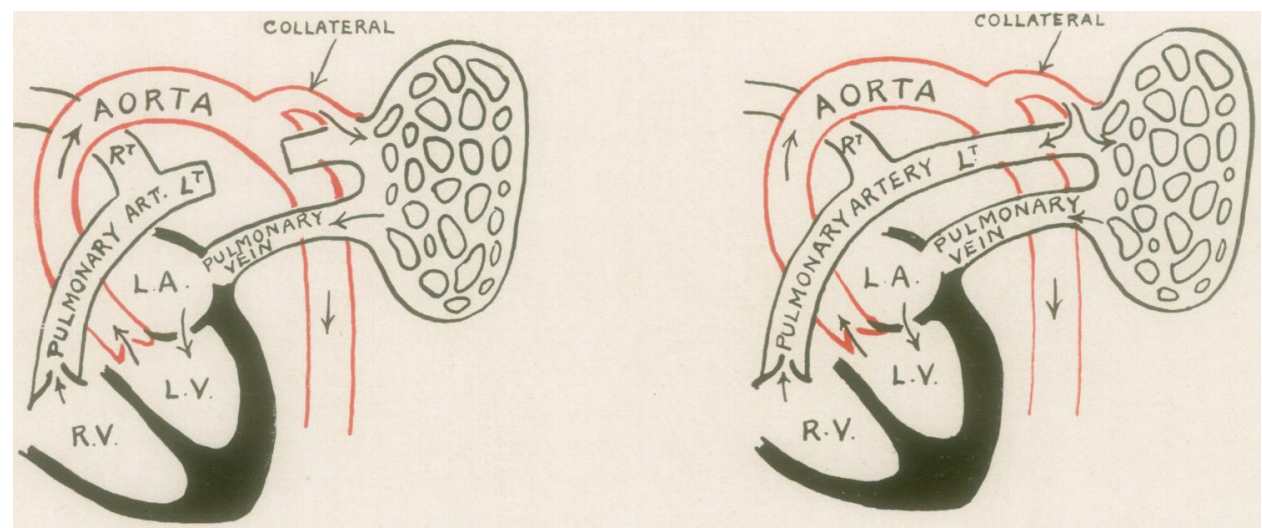

FIG. 2.-Circulation after ligation of pulmonary artery in dogs (left), and in bronchiectasis in man (right). 

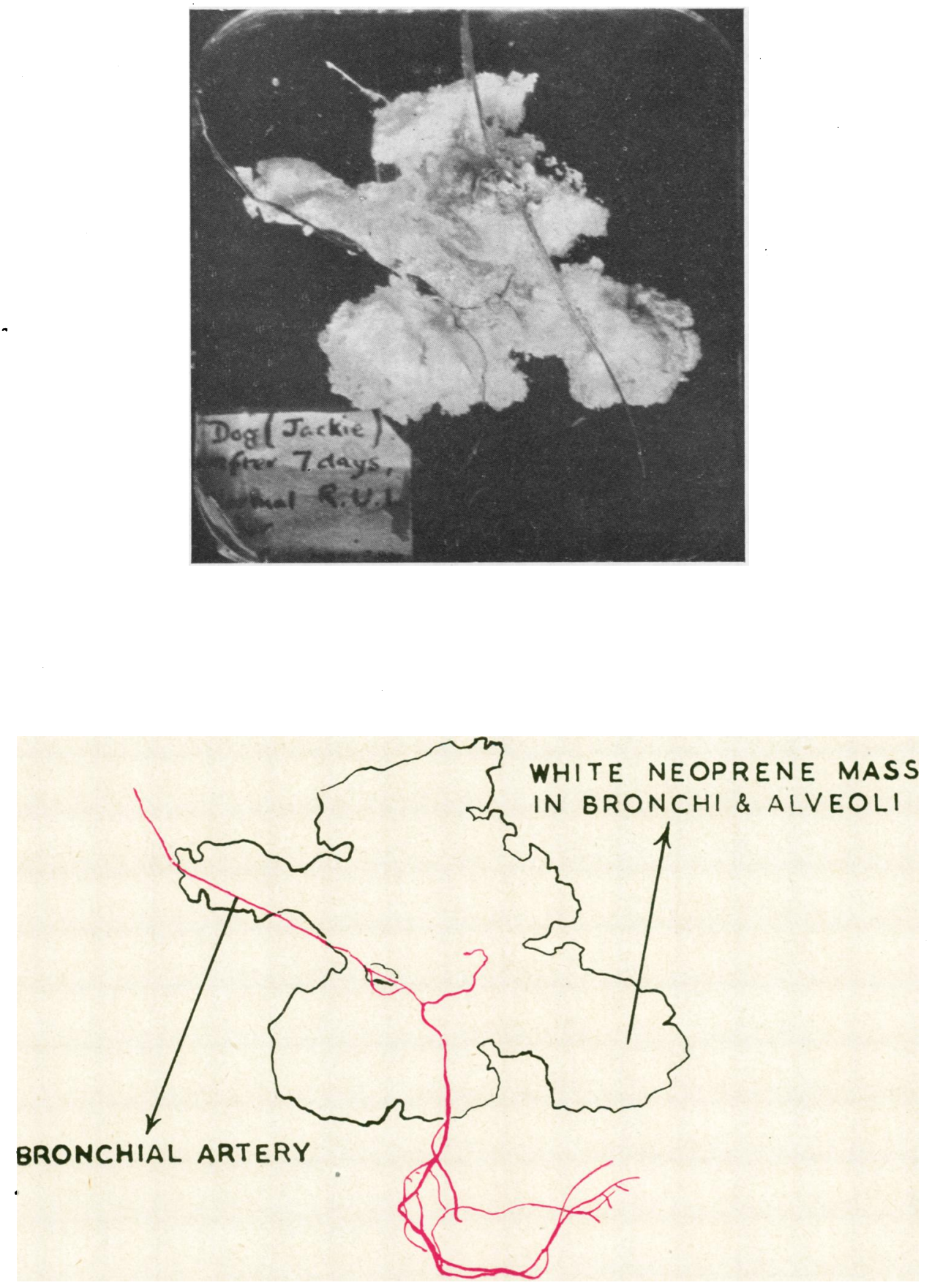

Plate 1.--Part of right upper lobe. Dog's lung. Normal 

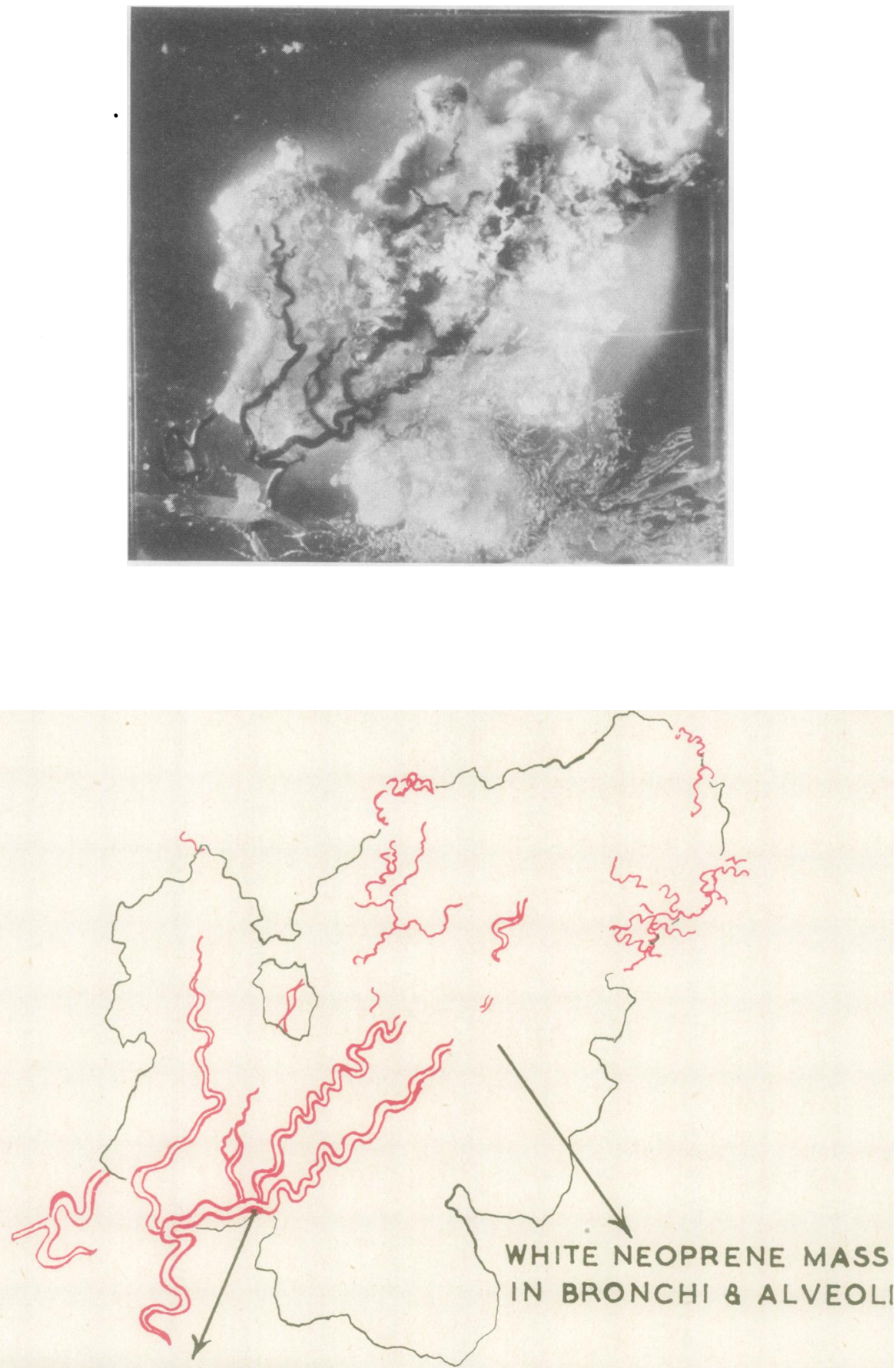

ENLARGED \& TORT UOUS

BRONCHIAL ARTERIES

Plate 2.- Left upper lobe. Same dog as Plate 1. Left pulmonary artery tied 7 days before death 
Plate 3.-This specimen shows the large tortuous bronchial arteries surrounding a straight and rather shrunken pulmonary artery. The pulmonary artery has been retrogradely injected from the enlarged bronchials, and thus the neoprene has entered the whole of the pulmonary vascular tree. Note also the branches from the intercostal arteries entering the lung.
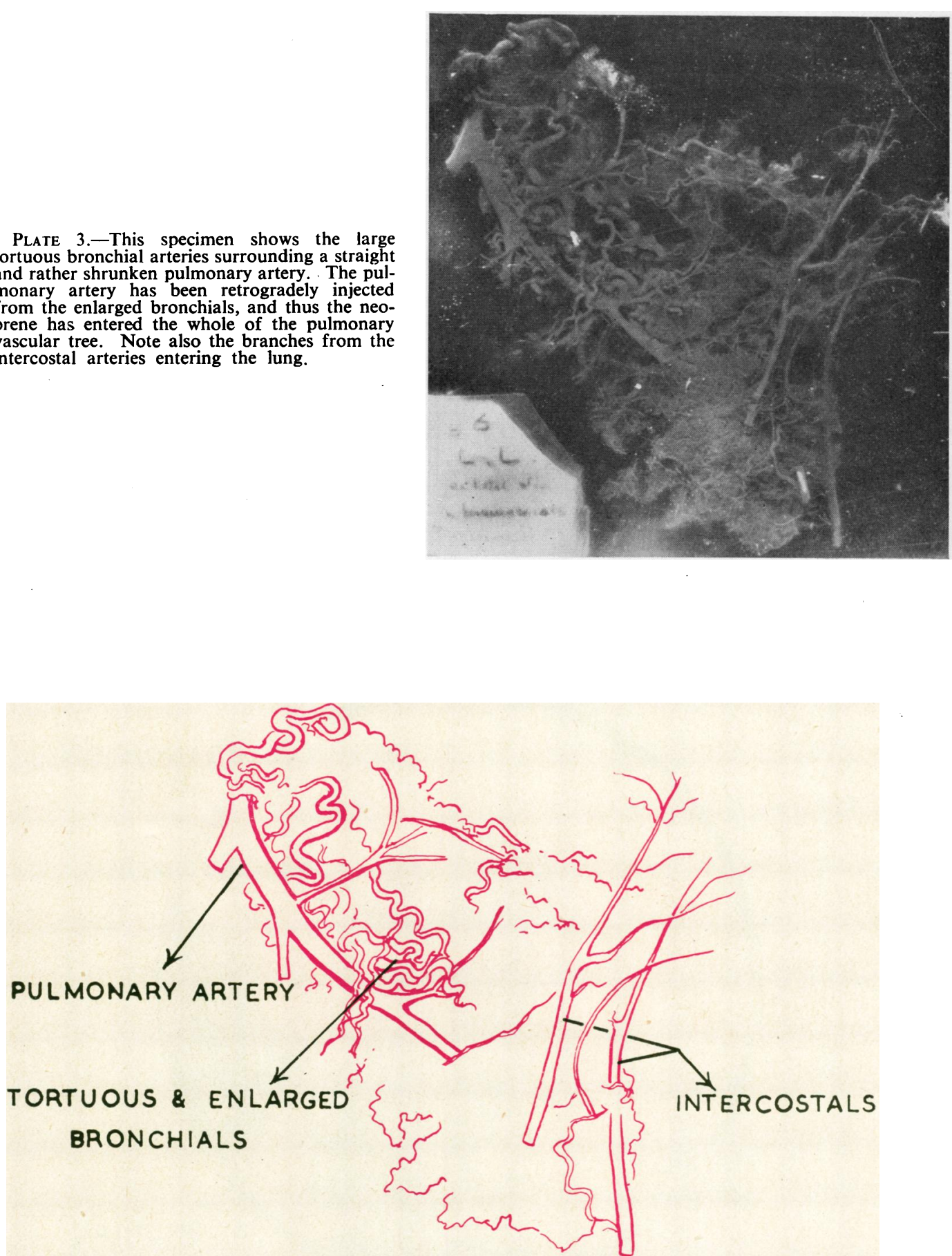

Left lower lobe. Dog's lung. Left pulmonary artery tied 14 months before death 


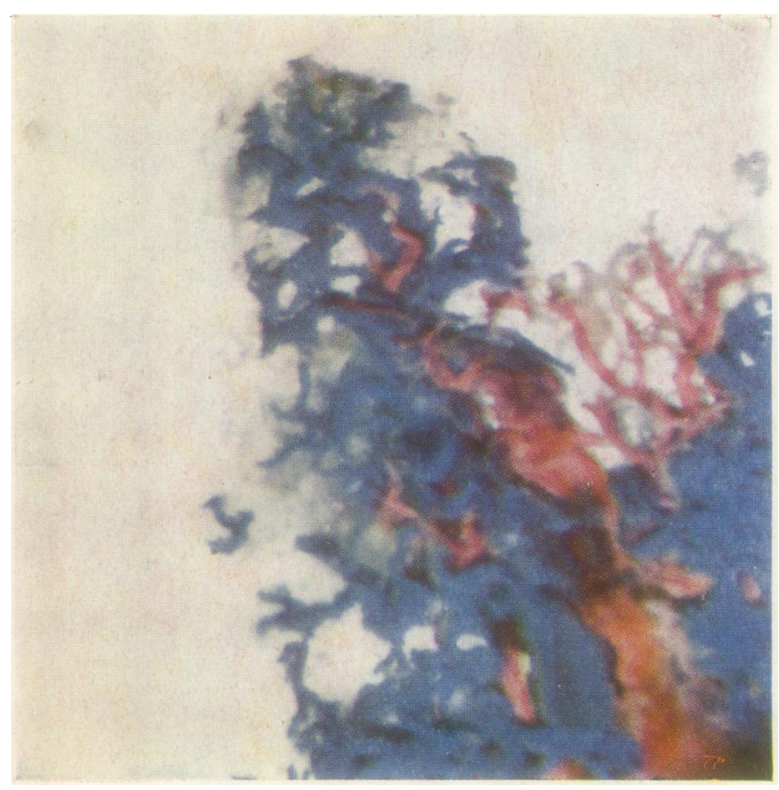

Plate 4--Part of the neoprene cast of the lung of a dog whose pulmonary artery had been tied nine months before death. A terminal lobule is shown $(\times 10)$ with the enlarged bronchial artery (red) accompanying the pulmonary artery (blue) right up to the base of the terminal lobular branch before it breaks up into the terminal capillary mesh. At the base of this lobular artery the terminal branch of the bronchial artery is seen anastomosing with it.

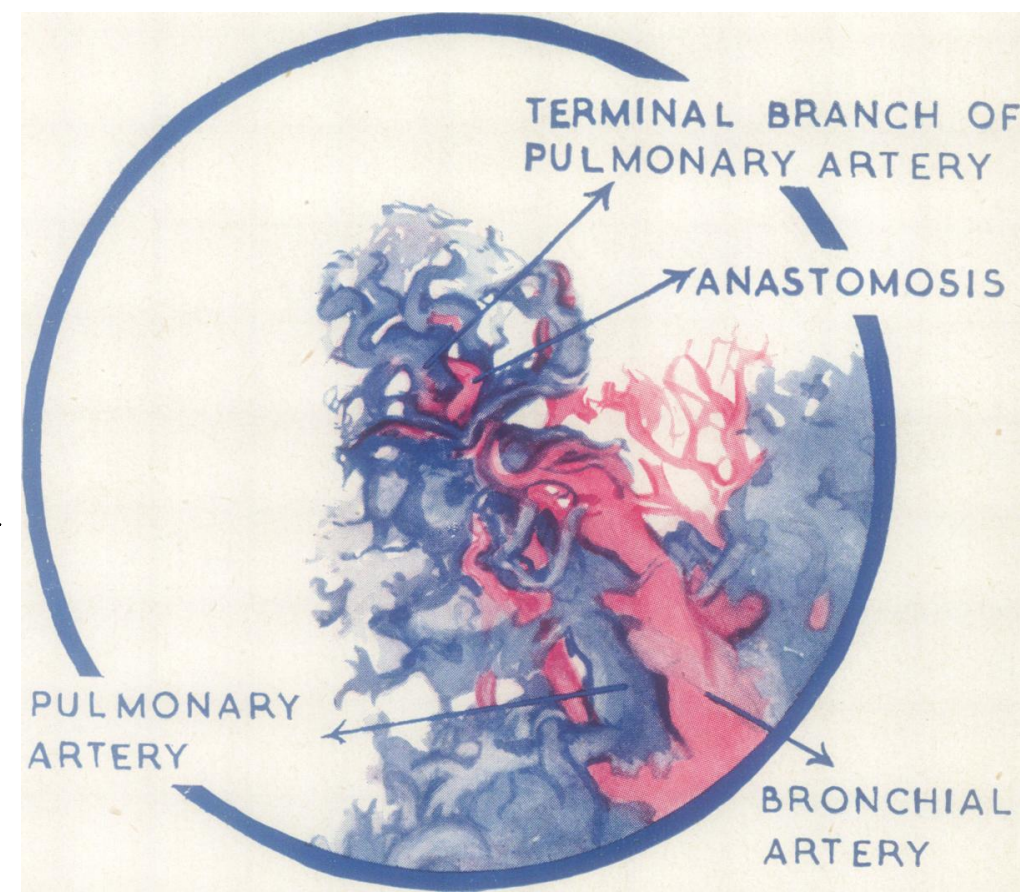

Terminal lobule. Dog’s lung 
Plate 5.-Neoprene cast of a small part of a human bronchiectatic lung. Red neoprene was injected into the bronchial artery, blue into the pulmonary artery, and white into the bronchus. The specimen has bcen flattened, and the pulmonary artery pulled over to one side to show the relations more clearly. The bronchiectatic sac is surrounded by a vast network of tortuous vessels derived from the bronchial arteries, among which several aneurysms may be seen. The pulmonary artery is shrunken and shows a mixture of red and white neoprene in its lumen. Large broncho-pulmonary anastomoses can be seen with the naked eye.
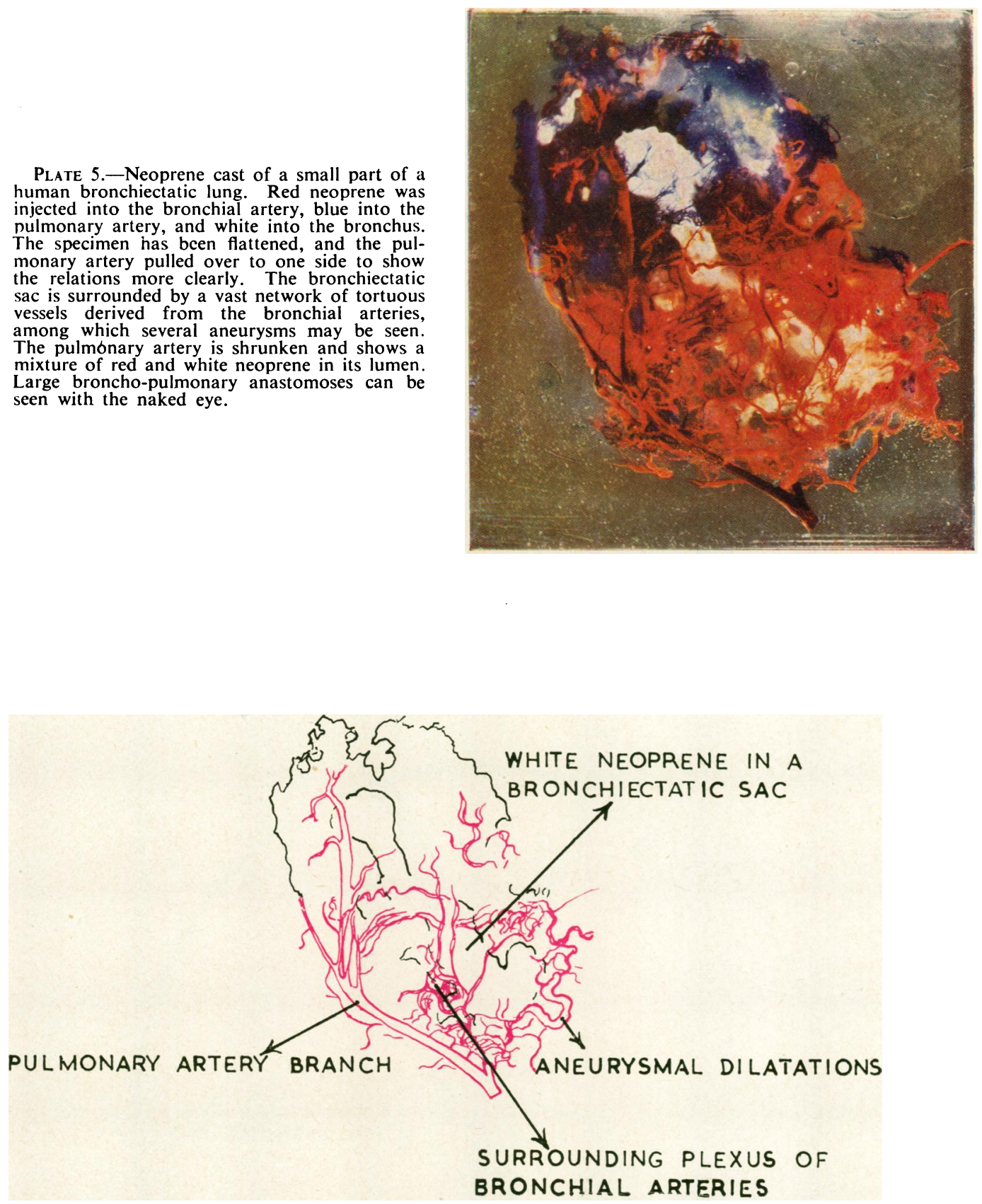

Part of left lower lobe. Human lung. Bronchiectasis. Operation specimen 


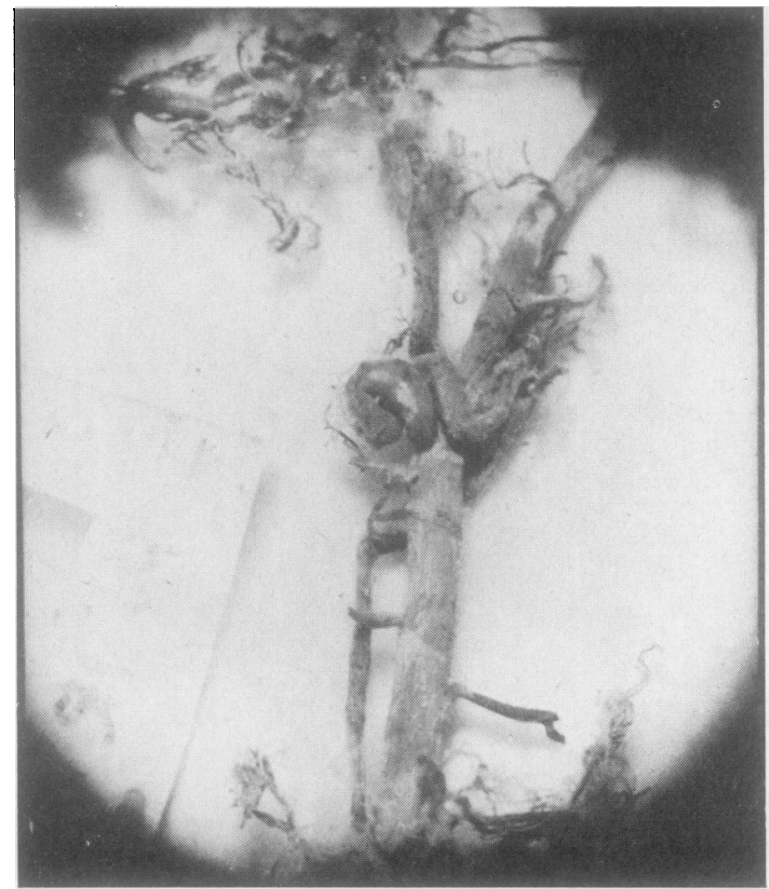

Plate 6.-This specimen shows one of the larger bronchopulmonary anastomoses. It occurs at the bifurcation of a large (4th order) pulmonary artery branch.

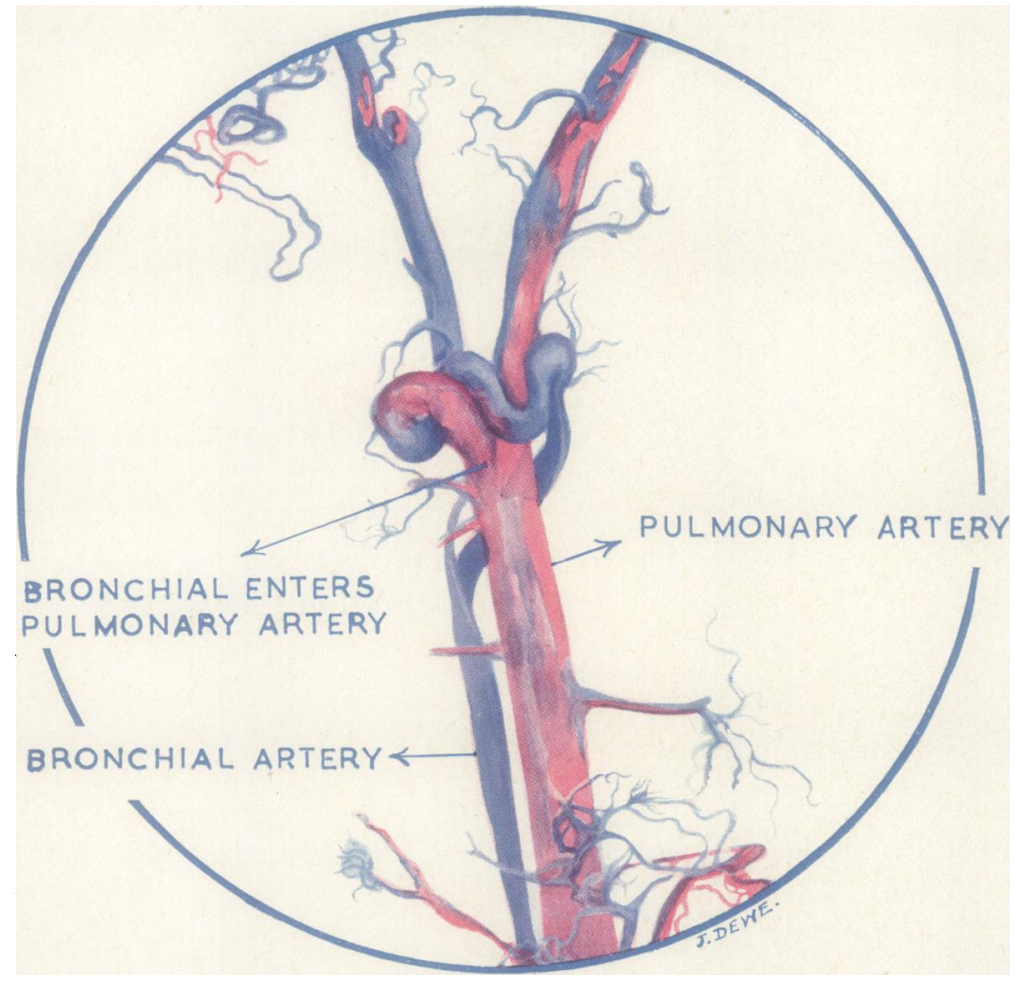

A broncho-pulmonary anastomosis in human bronchiectatic lung 

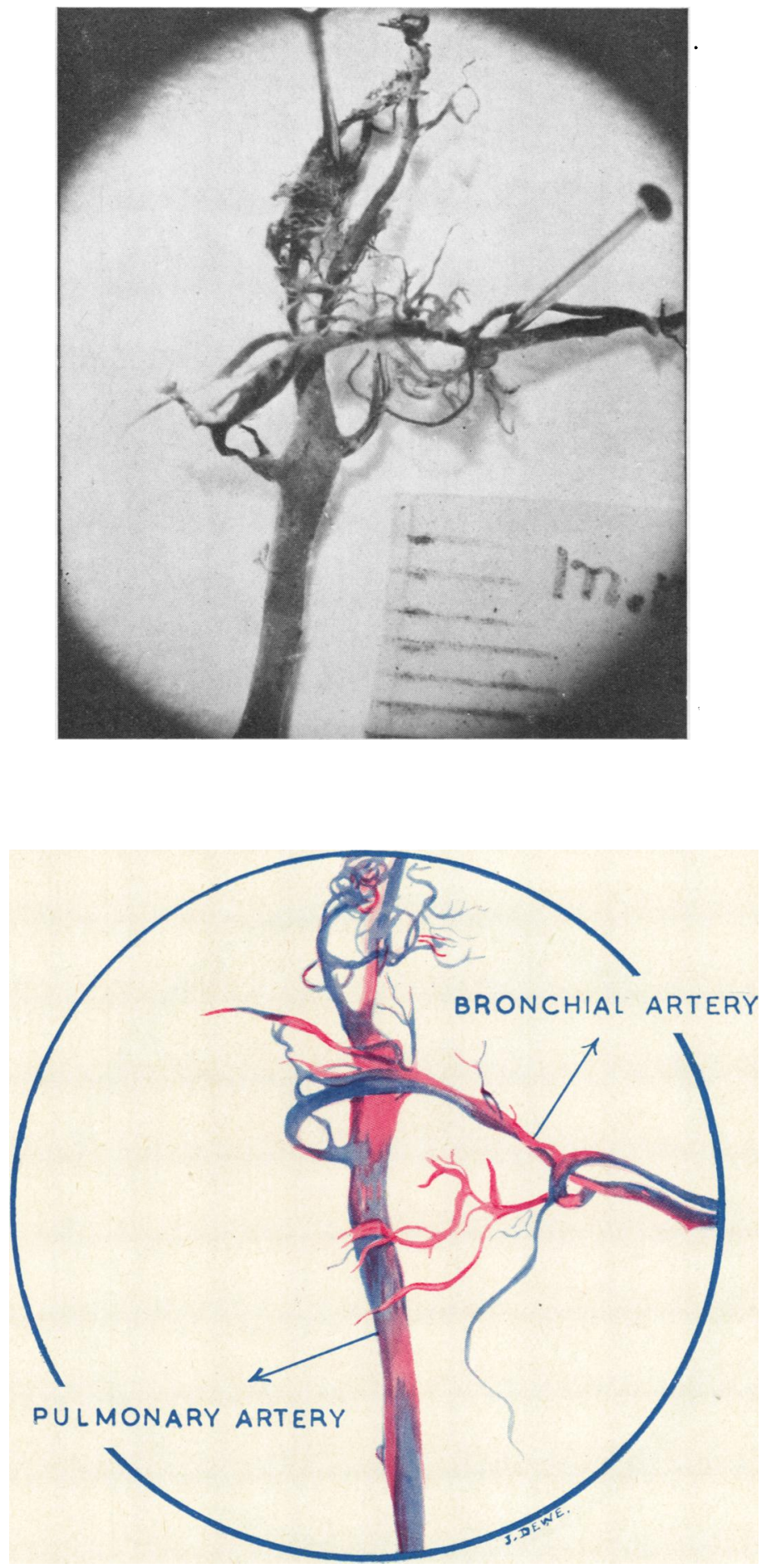

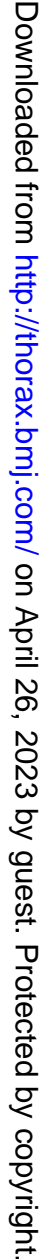

Plate 7.-- Another broncho-pulmonary anastomosis in human bronchiectatic lung 

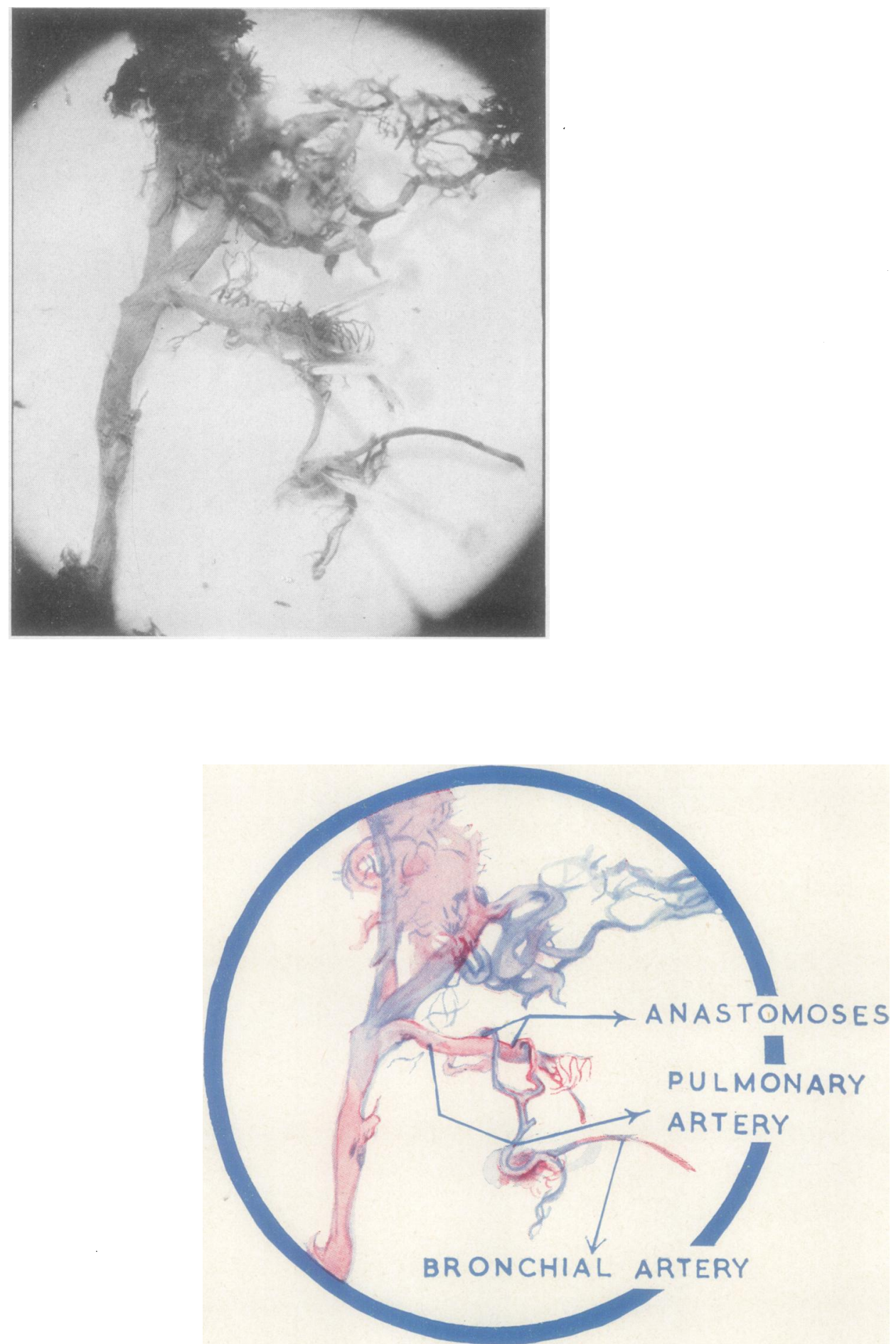

PLATE 8.-Smallest broncho-pulmonary anastomoses from wall of a bronchiectatic sac-human lung 
In the experimental dog, and in human cases of bronchiectasis and congenital heart disease, this broncho-pulmonary communication has been shown to be present and to be of considerable size. In cases of carcinoma and emphysema there is also evidence for its occurrence (Marchand, Gilroy, and Wilson, 1950).

The first question which arises is whether these communications are merely dilatations of already existing channels which are present, though small, in normal lungs or are communications which are formed de novo. We believe that they are dilatations of small channels which are present in normal lung. The existence of these minute communications in normal lung has been demonstrated by ourselves (Cockett and Vass, 1950), by Verloop (1948), and by Marchand et al. (1950). If the existence of these channels is granted, it would seem unnecessary to postulate new connexions developing.

Next, what is the stimulus to the bronchial collateral? In the experimental animal the most efficient stimulus is complete occlusion of the pulmonary artery. Cases of congenital heart disease with atresia or stenosis of the pulmonary arteries would come under the same heading. But in bronchiectasis the pulmonary arteries are patent. The theory has been put forward that at some time in the genesis of severe bronchiectasis the pulmonary arteries in the lung become thrombosed. They are then recanalized via the broncho-pulmonary anastomoses from the enlarged bronchial arteries which develop. There is histological evidence for this theory, in that a study of sections of bronchiectasis will often reveal large thrombosed arteries undergoing recanalization. If this is so, the thrombosis of pulmonary arteries would act as an adequate stimulus to the development of the bronchial collateral in the same way as ligation of the artery.

The effects of the bronchial collateral on the circulation through the lung is full of interesting possibilities which have already been most competently discussed by Liebow et al. (1950b). However, there are one or two points which may be emphasized. By means of the bronchopulmonary anastomoses a high-pressure system (the bronchial artery) is joined to a low-pressure system (the pulmonary artery). In a simple case of ligation of the pulmonary artery the course taken by the collateral flow is relatively easy to work out (Fig. 2); but when the pulmonary artery is not occluded, as in bronchiectasis, the possible effects are more complicated. A comparison of the two circulations is made by the two diagrams in Fig. 2.

In the case of the ligated pulmonary artery the collateral flow from the aorta passes into the pulmonary artery radicles within the lung, thence through the lung capillaries, and back to the left auricle via the pulmonary veins. Thus the whole of this collateral circulation is a burden on the left side of the heart, the output of the left ventricle exceeding that of the right by the amount of the collateral circulation. In dogs it has been shown by Liebow and his colleagues (Bloomer et al., 1949) that in these conditions the output of the left ventricle is one-third greater than that of the right.

In generalized bronchiectasis the collateral flow must be even greater than this. Also the collaterals flow into an unobstructed pulmonary artery. Thus the blood from the bronchial collateral might pass not only outwards through the lung capillaries but also retrogradely up the pulmonary artery. In any case it may have the effect of diverting most of the pulmonary artery blood away from the diseased side 
to the normal side. If this is so it might be thought that the pressure in the pulmonary artery of a bronchiectatic lung might be higher than that on the normal side. Great differences of pressure are not to be expected, as there is ample evidence that the capillary bed of the lung is adaptable to a surprising degree and can absorb enormous collateral flows with no change in pulmonary artery pressure (Cournand, 1947). No pulmonary hypertension would be expected unless the peripheral capillary bed of the lung was strikingly contracted by disease. Nevertheless, these bronchopulmonary anastomoses must represent points of increased resistance in the pulmonary artery, and a relatively small pressure gradient may be quite effective in diverting the pulmonary artery blood flow away from a diseased part.

The oxygen saturation of the blood in the distal part of the pulmonary artery should be considerably greater than that of mixed venous blood whenever the collateral develops, as it is receiving arterial blood via the anastomoses. In dogs the isolation of the distal part of the previously ligated pulmonary artery has proved an unexpectedly difficult manœuvre, and we have no reliable results yet. But Gilroy (1951) has found that in bronchiectasis the oxygen saturation of the blood from the pulmonary artery of the diseased side is greater than that of the normal side, which is in accordance with the anatomical findings described.

\section{SUMMARY}

The changes which take place in the bronchial arterial system after experimental ligation of the pulmonary artery in dogs, and in a human case of bronchiectasis, have been studied by means of neoprene bronchovascular casts.

The bronchial collateral is essentially similar in the two cases.

Pre-capillary bronchopulmonary anastomoses develop in both cases, but are larger and between bigger vessels in bronchiectasis.

The significance of these changes is discussed.

\section{FIXING MeTHOD}

Resin S.B.28C.* containing 5\% Monomer C. was used. This sets after a time at room temperature, giving a clear transparent casting with hard surfaces. Tanks made of glass slides bound together with adhesive tape were used as moulds to make the castings. After cleaning the glass with acetone no separating agent was required to assist the removal of the finished casts. The neoprene specimens were kept in water before mounting, and when needed were carefully surface-dried to avoid cloudiness. Dehydration through the alcohols was not necessary.

Our thanks are due to Mr. N. R. Barrett for his interest in the work throughout and for permission to publish details of the case under his care: to Roger Scholefield, our technician, for his assistance, and particularly for the mounting of the specimens in "marco-resin," and to A. L. Wooding for the photography of the specimens: to Miss Dewe for her painstaking illustrations, and to Mrs. Gowan for her secretarial work.

* "Marco-resin" is supplied by Scott Bader \& Co., Ltd., 109 Kingsway, W.C.2. Technical Leaflets 31 and 32. 


\section{REFERENCES}

Bloomer, W. E., Harrison, W., Lindskog, G. E., and Liebow, A. A. (1949). Amer. J. Physiol., $157,317$.

Cockett, F. B., and Vass, C. C. N. (1950). Brit. J. Surg., 38, 97.

Cournand, A. (1947). Bull. N.Y. Acad. Med., 23, 27.

Gilroy, J. C. (1951). Personal communication.

Liebow, A. A., Hales, M. R., Bloomer, W., Harrison, W., and Lindskog, G. E. (1950a). Amer. J. Path., 26, 177.

Hales, M. R., Harrison, W., Bloomer, W., and Lindskog, G. E. (1950b). Yale J. Biol. Med., 22, 637.

Marchand, P., Gilroy, J. C., and Wilson, V. H. (1950). Thorax, 5, 207.

Tait Smith (1951). Personal communication.

Verloop, M. C. (1948). Acta anat., Basel, 5, 171.

Wright, R. D. (1938). J. Path. Bact., 47, 489. 